\title{
Prestación de pastillas en el control de enfermedades de trigo
} Spray nozzles performance on wheat diseases control

\author{
Matilde Mur ${ }^{1}$, Santiago Gadea², Mariano Julio Ponce ${ }^{3}$, Víctor Hugo \\ Merani $^{4}$, Facundo Daniel Guilino ${ }^{5}$, Roberto Hernan Balbuena ${ }^{6}$, Juan \\ Manuel Vazquez ${ }^{7}$, Telmo Cecilio Palancar ${ }^{8}$
}

Mur, M; Gadea, S; Ponce, M; Merani, V; Guilino, F; Balbuena, R; Vasquez, J; Palancar, T. Prestación de pastillas en el control de enfermedades de trigo. Tecnología en Marcha. Vol. 32, Especial. XIII CLIA. Abril 2019. Pág 5-11.

DOI: https://doi.org/10.18845/tm.v32i7.4240

1 Ingeniero Agrónomo, Curso de Mecanización Agraria, Facultad de Ciencias Agrarias y Forestales, Universidad Nacional de La Plata, Argentina. Correo electrónico: matilde_mur@hotmail.com

2 Estudiante, Facultad de Ciencias Agrarias y Forestales, Universidad Nacional de La Plata, Argentina.

3 Ingeniero Agrónomo, Curso de Mecanización Agraria, Facultad de Ciencias Agrarias y Forestales, Universidad Nacional de La Plata, Argentina.

4 Ingeniero Agrónomo, Curso de Mecanización Agraria, Facultad de Ciencias Agrarias y Forestales, Universidad Nacional de La Plata, Argentina.

5 Ingeniero Agrónomo, Curso de Mecanización Agraria, Facultad de Ciencias Agrarias y Forestales, Universidad Nacional de La Plata, Argentina.

6 Ingeniero Agrónomo, Curso de Mecanización Agraria, Facultad de Ciencias Agrarias y Forestales, Universidad Nacional de La Plata, Argentina.

7 Ingeniero Agrónomo, Curso de Mecanización Agraria, Facultad de Ciencias Agrarias y Forestales, Universidad Nacional de La Plata, Argentina.

8 Ingeniero Agrónomo, Curso de Mecanización Agraria, Facultad de Ciencias Agrarias y Forestales, Universidad Nacional de La Plata, Argentina. 


\title{
Palabras clave
}

Cobertura; eficiencia; doble abanico; aire inducido.

\section{Resumen}

Las enfermedades fúngicas en el cultivo de trigo (Triticum aestivum) ocasionan pérdidas importantes en rendimiento y calidad de los granos. Se realizaron ensayos sobre un cultivo de trigo en antesis temprana (estado Z61), para evaluar la prestación de diferentes pastillas. El ensayo fue conducido en un esquema factorial de 4x2 con 10 repeticiones. Los factores fueron cuatro pastillas: abanico plano (XR11002), doble abanico asimétrico con aire inducido (Al307002), doble abanico simétrico con aire inducido (GAT11002), cono hueco (TXA8002), y dos objetivos artificiales, uno vertical representando la espiga y otro horizontal para la hoja bandera. Se utilizaron tarjetas hidrosensibles y el programa CIR $1.5^{\circledR}$ para la determinación de densidad de impactos (DI, imp $\mathrm{cm}^{-2}$ ), cobertura (COB, \%) y deposición (\%), siendo ésta contrastada con el uso de trazador Azul Brillante. Se realizó análisis de la varianza y las medias fueron comparadas por el test de Tukey $(p \leq 0,05)$. La pastilla TXA8002 alcanzó la mejor prestación, en DI, COB y deposición. AI3070 logró similar depósito que TXA8002 en la espiga, 51\% y $45 \%$ respectivamente, pese a tener la menor DI, (44 imp cm-2), mientras que en hoja bandera TXA8002 tuvo el mayor porcentaje de depósito (40\%). Las pastillas con aire inducido tuvieron la menor DI pero una mayor uniformidad entre las distintas caras de la espiga. Las metodologías de evaluación utilizadas resultaron complementarias para realizar un análisis completo de caracterización de la aplicación.

\section{Keywords}

Coverage; efficiency; twin nozzles; air induction.

\begin{abstract}
Fungal diseases in wheat crops (Triticum aestivum) cause significant yield and quality grain losses worldwide. Field trials were carried out at the beginning of anthesis on wheat crop (state Z61), to evaluate different spray nozzles performance. The test was arranged in a 4x2 factorial design with ten repetitions. Factors consisted in four nozzles: flat-fan (XR 11002), air induction asimetrical dual flat fan (Al3070-02), air induction simetrical dual flat fan (GAT11002), hollow cone (TXA8002), and two artificial targets, one vertical and another horizontal related to head and flag leaf location, respectively. Water-sensitive cards and CIR $1.5^{\circledR}$ software were used for their evaluation, determining droplet density (DD, drop $\left.\mathrm{cm}^{-2}\right)$, coverage (\%) and deposition (\%), being the latter contrasted with the use of Brilliant Blue tracer. Data were subjected to ANOVA and means were compared by Tukey test $(p \leq 0,05)$. The TXA8002 reached the best performance in DD, coverage, and deposition. Al3070 achieved similar deposition than TXA8002 in head, $51 \%$ and $45 \%$ respectively, despite having the lowest DD (44 drop $\mathrm{cm}^{-2}$ ), while TXA8002 had the higher deposition on flag leaf (40\%). Air induction nozzles had the lowest DD but greater uniformity between head sides. The evaluation methodologies used are complementary when a complete analysis of application parameters is looking for.
\end{abstract}




\section{Introducción}

El trigo (Triticum aestivum) es el cereal de invierno más importante de la Argentina. La presencia de enfermedades foliares y de la espiga provocan grandes reducciones en el rendimiento y en la calidad de los granos. Para lograr disminuir el desarrollo de las mismas, se hace hincapié en la aplicación de fungicidas en el momento oportuno. En el caso de la fusariosis de la espiga, la aplicación debe ser en forma preventiva, con productos fungicidas que actúen principalmente como de contacto, por lo cual deben ser aplicados inmediatamente antes de la ocurrencia de la infección. Se recomienda para aplicaciones de fungicidas de contacto una cobertura mínima de 60 gotas por $\mathrm{cm}^{2}$ [1]. Sin embargo, además de la densidad de impactos, también es importante el diámetro de los mismos para este tipo de productos. Se han obtenido mejores porcentajes de cobertura, tanto en la espiga como en la hoja bandera pulverizando con gotas finas [2]. El tipo de boquilla afecta significativamente la deposición tanto en la cara frontal, como en la trasera y en todo el objetivo [3]. En este sentido, las boquillas de doble abanico plano presentaron mayor uniformidad de distribución sobre las espigas que las boquillas de abanico plano convencional, determinando una mayor cobertura y control de la enfermedad [4]. Evaluando pastillas simétricas y asimétricas, se ha determinado una mayor densidad de impactos y cobertura con boquillas anguladas en $30^{\circ}$ hacia adelante y hacia atrás [5].

En razón de lo expuesto, los resultados son variables y complejos, lo que hace importante la evaluación de las aplicaciones de fitoterápicos. El uso de tarjetas hidrosensibles es una metodología accesible y simple que permite la valoración de distintos parámetros que inciden sobre la eficiencia de control. En los últimos años se han desarrollado diferentes formas de procesamiento digital de tarjetas, que permiten cuantificar características de la población de gotas y la cantidad de producto depositado, que se expresa como eficiencia. Al respecto, comparando pastillas convencionales y de aire inducido, se ha encontrado que la metodología de procesamiento de imágenes sobreestima la eficiencia de aplicación para pastillas con inducción de aire principalmente [6]. El presente trabajo tiene por objetivos evaluar la calidad y uniformidad de aplicación de diferentes tipos de boquillas sobre un cultivo de trigo y determinar la deposición de las mismas calculada a partir de la metodología de digitalización de imágenes y la determinación analítica por colorimetría.

\section{Materiales y métodos}

Los ensayos se realizaron sobre un cultivo de trigo (Triticum aestivum) en antesis temprana. Se utilizó un equipo pulverizador hidráulico con un ancho de $8,4 \mathrm{~m}$, portapicos distanciados a 0,525 m y altura de botalón respecto a la espiga de 0,38 m. La velocidad de desplazamiento fue de 2,45 $\mathrm{m} \mathrm{s}^{-1}$, la presión de trabajo de $300 \mathrm{kPa}$ y la tasa de aplicación de 103 I ha-1. Para las aplicaciones se utilizó agua y un trazador colorimétrico Azul Brillante en una dilución de $13,073 \mathrm{~g} \mathrm{l}^{-1}$. Las condiciones meteorológicas promedio durante el ensayo fueron: temperatura $26^{\circ} \mathrm{C}$, humedad relativa $41 \%$ y velocidad del viento de $5 \mathrm{~km} \mathrm{~h}^{-1}$ con ráfagas de $11,3 \mathrm{~km} \mathrm{~h}^{-1}$. Se utilizó un diseño factorial de 4x2 con diez repeticiones distribuidas al azar. Se evaluaron cuatro diseños de pastillas, abanico plano $11002(\mathrm{AP})$, cono hueco $8002(\mathrm{CH})$, doble abanico simétrico con aire inducido 11002 (Al 3030) y doble abanico asimétrico con aire inducido 11002 (Al 3070) que definieron los tratamientos y se establecieron dos objetivos de medición: uno vertical, la espiga; y otro horizontal, la hoja bandera. Los objetivos verticales fueron evaluados con dos metodologías. Por un lado, luego de realizada la aplicación, se recolectaron diez espigas y se lavaron con agua destilada. Con el residuo obtenido se realizó la lectura de color mediante un espectrofotómetro y a partir de curvas de calibración se determinó la cantidad de líquido recolectado en cada una. Por otro lado, mediante un tubo apoyado sobre la espiga, se colocaron cuatro tarjetas hidrosensibles simulando las caras de la misma: anterior, posterior, derecha e 
izquierda. Esta última metodología fue también utilizada para valorar los objetivos horizontales, colocando papeles sensibles sobre un soporte de hierro a la altura de la hoja bandera. Las tarjetas fueron digitalizadas a 1200 dpi y analizadas con el software de imágenes CIR 1.5®, determinando: 1) Densidad de impactos (DI, imp. $\mathrm{cm}^{-2}$; 2) Cobertura (COB, \%) y 3) Deposición (\%) referida como la relación entre el volumen recogido en el objetivo y el aplicado por la pulverizadora. Sobre los datos relevados se efectuó un análisis factorial siendo la significancia de las diferencias establecidas por el test de Tukey $(\mathrm{p} \leq 0,05)$.

\section{Resultados y discusión}

Tanto en espiga como en hoja bandera la boquilla $\mathrm{CH}$ presentó la mayor DI y las de doble abanico la menor (cuadro 1), atribuyendo estas diferencias al espectro de gotas de menor tamaño de las boquillas $\mathrm{CH}$, con mayor probabilidad de depositarse que las gotas más grandes. Todos los tratamientos presentaron mayor DI sobre la hoja bandera respecto a la espiga, corroborando las dificultades de las gotas para alcanzar el objetivo vertical, vinculadas a la propia estructura de la espiga y su orientación. Tomando la recomendación de [1], de $60 \mathrm{imp} \mathrm{cm}^{-2}$, a excepción de la boquilla AI3070, todas alcanzaron en la espiga la DI necesarios.

Cuadro 1. Densidad de impactos (DI) y \% de Cobertura (COB) sobre espiga y hoja bandera para AP: abanico plano 11002; CH: cono hueco TXA 8002; AI3030: doble abanico simétrico con aire inducido 11002 y Al3070: doble abanico asimétrico con aire inducido 11002. Letras minúsculas distintas indican diferencias significativas $(p \leq 0,05)$ según el test de Tukey entre boquillas para cada objetivo y cada variable respuesta. Letras mayúsculas distintas indican diferencias significativas $(p \leq 0,05)$ según el test de Tukey entre objetivos para cada variable respuesta.

\begin{tabular}{|c|c|c|c|c|c|c|c|c|c|}
\hline \multicolumn{5}{|c|}{ DI (gotas $\left.\mathrm{cm}^{-2}\right)$} & \multicolumn{5}{|c|}{$\% \mathrm{COB}$} \\
\hline Pastillas & \multicolumn{2}{|c|}{ Espiga } & \multicolumn{2}{|c|}{ Hoja Bandera } & Pastillas & \multicolumn{2}{|c|}{ Espiga } & \multicolumn{2}{|c|}{ Hoja Bandera } \\
\hline Al 3070 & $44,4 \mathrm{a}$ & \multirow{4}{*}{ A } & 80,78 a & \multirow{4}{*}{ B } & Al 3070 & $4,6 \mathrm{a}$ & \multirow{4}{*}{ A } & $15,76 \mathrm{a}$ & \multirow{4}{*}{ B } \\
\hline AP & 89,58 a & & $195,9 b$ & & AP & $5,3 \mathrm{a}$ & & $16,51 \mathrm{a}$ & \\
\hline Al 3030 & $64,8 \mathrm{a}$ & & 91,9 a & & Al 3030 & $4,4 \mathrm{a}$ & & $12 \mathrm{a}$ & \\
\hline $\mathrm{CH}$ & 147,33 b & & $277,9 \mathrm{c}$ & & $\mathrm{CH}$ & $5,8 \mathrm{a}$ & & $15,78 \mathrm{a}$ & \\
\hline
\end{tabular}

En el análisis de la DI en las caras de la espiga (figura 1), CH, Al3030 y Al3070 lograron la mayor cantidad sobre la cara posterior, aunque solo $\mathrm{CH}$ se diferenció significativamente. La angulación hacia atrás de las pastillas doble abanico, en $30^{\circ}$ para Al3030 y $70^{\circ}$ para Al3070, permite que el chorro asperjado sea dirigido hacia la parte posterior principalmente. Asimismo, el tamaño de gota reducido y el efecto de rotación del flujo en $360^{\circ}$ de $\mathrm{CH}$ determinan que las gotas se muevan en múltiples direcciones alcanzando a la espiga en toda su superficie. En cambio, el comportamiento de AP puede deberse a la posición de la boquilla respecto al botalón, sin angulación, definiendo una cortina de pulverizado con mayor incidencia en los laterales. En la valoración de la uniformidad de distribución, las boquillas de doble abanico presentaron mayor uniformidad respecto a $\mathrm{CH}$ y $\mathrm{AP}$, en acuerdo con [4]. $\mathrm{CH}$ fue la que mayor desuniformidad presentó, mientras que AP tuvo un comportamiento intermedio atribuible, en principio, al perfil de distribución triangular, sin grandes depresiones en la zona central. 


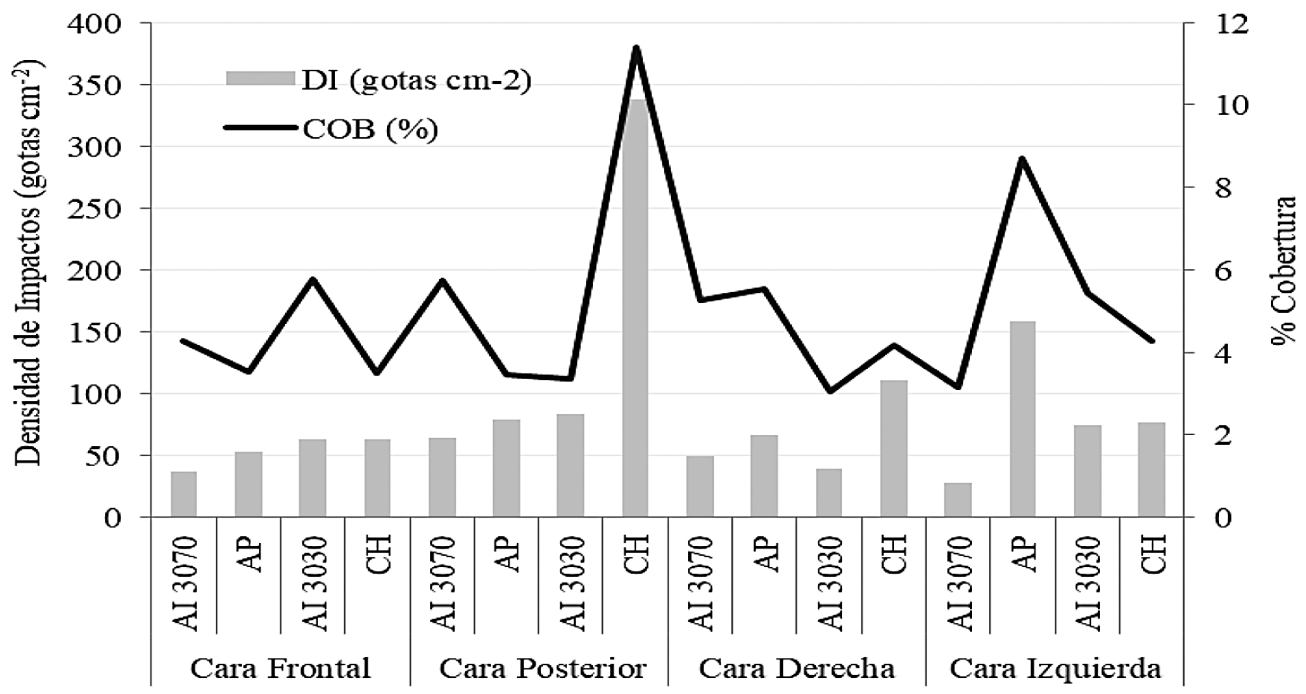

Figura 1. Densidad de impactos (DI) y \% de Cobertura (COB) sobre las caras de la espiga para AP: abanico plano 11002; CH: cono hueco TXA 8002; Al3030: doble abanico simétrico con aire inducido 11002 y Al3070: doble abanico asimétrico con aire inducido 11002.

En cuanto a la COB, las tendencias resultaron semejantes a las de DI, con los mayores porcentajes sobre la hoja bandera, con diferencias significativas respecto a la espiga para todas las boquillas. En la espiga, los porcentajes de COB no superaron el 6\%, mientras que en la hoja bandera entre el $12 \%$ y $16 \%$ aproximadamente. Los bajos porcentajes sobre las espigas, ponen en riesgo el control de la Fusariosis, siendo que los productos actúan principalmente por contacto sobre el filamento de la antera. Tanto en el objetivo vertical como en el horizontal, las boquillas tuvieron similar comportamiento, $\mathrm{CH}$ y AP lograron porcentajes apenas superior a las boquillas anguladas, sin diferencias significativas, en acuerdo con [2] quienes obtuvieron los mejores porcentajes de COB con gotas finas, representativas de AP y $\mathrm{CH}$. Las boquillas doble abanico, tuvieron similar prestación en la espiga pero no en la hoja bandera, donde Al3030 tuvo la menor COB, en contraposición a lo observado por [5].

En el análisis de la COB sobre las caras de la espiga, $\mathrm{CH}$, Al3070 y AP presentaron los mayores porcentajes sobre la cara posterior en correspondencia con el número de impactos, pero en Al3030, fue la cara frontal la de mayor COB asociado esto a un mayor diámetro de las gotas que alcanzaron esa superficie. Los menores porcentajes de COB con las boquillas doble abanico, se observaron en los laterales de la espiga, pudiendo deberse a los ángulos de las mismas que producen un asperjado direccionado principalmente hacia las caras anterior y posterior. Hacia los laterales, la cantidad de líquido pulverizado disminuye en acuerdo con el perfil característico del abanico y la mayor distancia hacia el objetivo incide en la trayectoria de las gotas de los extremos que tienden a caer en sentido vertical a medida que se alejan. Mientras que, para las boquillas convencionales los menores porcentajes estuvieron en la cara frontal. No obstante estas consideraciones, las boquillas mantuvieron la uniformidad que se observó en la variable DI.

En lo referente a la recuperación del producto sobre la espiga, el software CIR 1.5 arrojó mayores valores, siendo la diferencia respecto al trazador significativa (cuadro 2). Estos resultados permiten confirmar que el programa sobreestima la eficiencia de aplicación en acuerdo con [6]. Asimismo, con la metodología de procesamiento de imágenes, los resultados presentaron gran variabilidad, sin diferencias significativas entre boquillas. Por lo contrario, 
con el uso del trazador, las boquillas se diferenciaron significativamente y los resultados fueron altamente estables, lo que indica en parte la confiabilidad de la metodología. En este sentido, los resultados tuvieron similar tendencia a los de cobertura, $\mathrm{CH}$ presentó la mayor deposición, significativamente diferente a AP y Al3030, pero con similar comportamiento a Al3070. En el análisis comparativo de las pastillas doble abanico respecto a AP, la inclinación en $30^{\circ}$ de Al3030 no fue suficiente para mejorar los depósitos, siendo necesaria una mayor angulación. De los resultados se desprende que el tipo de boquilla afecta significativamente la deposición en el objetivo, en coincidencia con [3]. La combinación de una pulverización dirigida hacia el objetivo junto con un tamaño de gota reducido, pareciera ser la mejor alternativa para una mayor eficiencia sobre la espiga, en condiciones atmosféricas ideales.

Cuadro 2. Depósito de caldo sobre la espiga según metodología de valoración y coeficiente de corrección. CIR 1.5: programa de procesamiento de imágenes; T: trazador. AP: abanico plano 11002; CH: cono hueco TXA 8002; Al3030: doble abanico simétrico con aire inducido 11002; Al3070: doble abanico asimétrico con aire inducido 11002. Letras minúsculas diferentes indican diferencias significativas $(p \leq 0,05)$ según el test de Tukey entre boquillas para cada metodología. Letras mayúsculas diferentes indican diferencias significativas $(p \leq 0,05)$ según el test de Tukey entre metodologías.

\begin{tabular}{|c|c|c|c|c|c|}
\hline Pastillas & \multicolumn{2}{|c|}{ CIR 1.5} & \multicolumn{2}{|c|}{ Trazador (T) } & Coeficiente de corrección (CIR 1.5/T) \\
\hline $\mathrm{CH}$ & 63,06 a & \multirow{4}{*}{ B } & $51,01 \mathrm{c}$ & \multirow{4}{*}{ A } & 1,24 \\
\hline Al 3030 & $63,74 \mathrm{a}$ & & 20,81 a & & 3,06 \\
\hline Al 3070 & 91,36 a & & $44,92 \mathrm{bc}$ & & 2,03 \\
\hline AP & $70,85 \mathrm{a}$ & & $35,89 \mathrm{~b}$ & & 1,97 \\
\hline
\end{tabular}

Los coeficientes de corrección obtenidos a partir de la relación de solución recuperada entre las dos metodologías (cuadro 2), fueron mayores para las boquillas de aire inducido, mientras que para $\mathrm{CH}$ y AP fueron menores. Estos resultados, corroboran la sobreestimación de la metodología de análisis digital de tarjetas hidrosensibles, siendo la sobrevaloración mayor para las inducidas con aire respecto de las convencionales, en acuerdo con [6]. Estas diferencias podrían ser explicadas por las características de las gotas con aire inducido, que impactan y dejan manchas en relación a su volumen y no de la real cantidad de agua que contienen. Además, al golpear en la superficie de la tarjeta la misma se rompe y genera nuevas gotas con un volumen que es estimado en forma repetida [6]. En cuanto a las gotas de menor tamaño, la menor sobreestimación estaría relacionada con la menor masa y menor arrastre de las mismas, además de las limitaciones del papel hidrosensible en detectar impactos de tamaño reducido. Afectando los valores de eficiencia con los coeficientes (cuadro 3), se encontró similar tendencia en ambos objetivos, siendo $\mathrm{CH}$ la de mayores eficiencias, con diferencias significativas respecto a Al3030. No obstante, las eficiencias fueron relativamente bajas, no superando el 50 \% en la espiga, mientras que en la hoja bandera, solo $\mathrm{CH}$ alcanzó el $40 \%$ de producto depositado.

La distribución del asperjado sobre la espiga, presentó diferentes comportamientos. $\mathrm{CH}$ fue la más desuniforme, debido a la gran deposición en la cara posterior respecto a las demás. En tanto que, las boquillas de doble abanico presentaron una mayor uniformidad, con una variación entre caras del 9\% a 13\%. Es claro que las direcciones múltiples son una mejora en general con respecto a las pastillas convencionales en la uniformidad de distribución más que en la cantidad de depósitos sobre el objetivo. 
Cuadro 3. Depósito de caldo recogido sobre la espiga y hoja bandera luego de ser afectado por el coeficiente. AP: abanico plano 11002; CH: cono hueco TXA 8002; Al3030: doble abanico simétrico con aire inducido 11002; Al3070: doble abanico asimétrico con aire inducido 11002. Letras minúsculas distintas indican diferencias significativas $(p \leq 0,05)$ según el test de Tukey entre boquillas para cada objetivo. Letras mayúsculas diferentes indican diferencias significativas $(p \leq 0,05)$ según el test de Tukey entre objetivos para cada boquilla.

\begin{tabular}{|c|c|c|c|c|c|}
\hline Objetivos de aplicación & Pastillas & \% Depósito & Objetivo de aplicación & Pastillas & \% Depósito \\
\hline \multirow{4}{*}{$\begin{array}{l}\frac{\tilde{\sigma}}{.} \\
\frac{0}{0} \\
\text { 山 }\end{array}$} & Al 3070 & $45,01 \mathrm{~b} \mathrm{~A}$ & \multirow{4}{*}{ 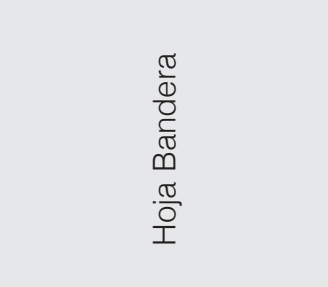 } & Al 3070 & $26,18 \mathrm{ab} A$ \\
\hline & AP & $35,96 \mathrm{ab} A$ & & AP & $31,27 \mathrm{ab} B$ \\
\hline & Al 3030 & 20,83 a $A$ & & Al 3030 & 17,01 a $A$ \\
\hline & $\mathrm{CH}$ & $50,85 \mathrm{~b} \mathrm{~A}$ & & $\mathrm{CH}$ & $40,47 \mathrm{~b} \mathrm{~A}$ \\
\hline
\end{tabular}

\section{Conclusiones}

La inclinación en $70^{\circ}$ hacia atrás, mejora la COB y la deposición sobre la espiga respecto a ángulos menores. Sin embargo, inclinaciones de $30^{\circ}$ hacia adelante no mejoran la penetración respecto a la posición vertical. La utilización de gotas finas y direcciones múltiples aumentan la COB y la deposición en objetivos verticales y horizontales, mientras las condiciones atmosféricas lo permitan. Las metodologías de evaluación utilizadas presentan ventajas y desventajas que son complementarias al momento de realizar un análisis completo de caracterización de la aplicación.

\section{Referencias}

[1] M. A. Gandolfo, J. Bueno, J. Torres Pereira, W. Sánchez, B. F. Zanni, y R. B. Belani. (2007). Avaliação da qualidade da aplicação com diferentes pontas de pulverização e diferentes volumes de aplicação na soja. Anais I Simpósio em Engenharia Rural (43-47). Universidade Estadual do Paraná Bandeirantes, Brasil.

[2] R. C. Derksen, P. A. Paul, H. E. Ozkan y H. Zhu. (2012). Field evaluations of application techniques for fungicide spray deposition on wheat and artificial targets. Applied Enineering in Agriculture. 28(2): 325-331.

[3] T. M. Wolf y B. C. Caldwell. (2013). Spray Deposition of TeeJet Al3070VS on Vertical Targets. Agriculture \& Agri-Food Canada.

[4] P. Nicholson, J. Turner, P. Jenkinson, P. Jennings, J. Stonehouse, M. Nuttall, D. Dring, G. Weston y M. Thomsett. (2003). Maximising control with fungicides of Fusarium ear blight (FEB) in order to reduce toxin contamination of wheat. Recuperado de: http://www.hgca.com.

[5] J. C. Ferguson, A. J. Hewitt y C. C. O’Donell. (2016). Pressure, droplet size classification, and nozzle arrangement effects on coverage and droplet number density using air-inclusion dual fan nozzles for pesticide applications. Crop Protection 89: 231-238.

[6] G. N. Domper, R. H. Balbuena y M. Mur. (2015). Eficiencia de aplicación de pastillas de pulverización con inducción de aire en el cultivo de soja. Recuperado de: http://www.agro.unlp.edu.ar/revista/index.php/revagro/ article/view/280/171. 